\title{
Imaging of the dynamic magnetic structure in a parallel array of shunted Josephson junctions
}

\author{
Doderer, T.; Kaplunenko, V. K.; Mygind, Jesper; Pedersen, Niels Falsig
}

Published in:

Physical Review B

Link to article, DOI:

10.1103/PhysRevB.50.7211

Publication date:

1994

Document Version

Publisher's PDF, also known as Version of record

Link back to DTU Orbit

Citation (APA):

Doderer, T., Kaplunenko, V. K., Mygind, J., \& Pedersen, N. F. (1994). Imaging of the dynamic magnetic structure in a parallel array of shunted Josephson junctions. Physical Review B, 50(10), 7211-7214.

https://doi.org/10.1103/PhysRevB.50.7211

\section{General rights}

Copyright and moral rights for the publications made accessible in the public portal are retained by the authors and/or other copyright owners and it is a condition of accessing publications that users recognise and abide by the legal requirements associated with these rights.

- Users may download and print one copy of any publication from the public portal for the purpose of private study or research.

- You may not further distribute the material or use it for any profit-making activity or commercial gain

- You may freely distribute the URL identifying the publication in the public portal 


\title{
Imaging of the dynamic magnetic structure in a parallel array of shunted Josephson junctions
}

\author{
T. Doderer \\ Physikalisches Institut, Lehrstuhl Experimentalphysik II, Universität Tübingen, D-72076 Tübingen, Germany \\ V. K. Kaplunenko \\ Institute of Radio Engineering and Electronics, Russian Academy of Sciences, 103907 Moscow, Russia \\ J. Mygind and N. F. Pedersen \\ Physics Department, Technical University of Denmark, DK-2800 Lyngby, Denmark
}

(Received 10 June 1994)

\begin{abstract}
A one-dimensional (1D) parallel array of shunted Josephson junctions is one of the basic elements in the family of rapid single-flux quantum logic circuits. It was found recently that current steps always show up in the current-voltage curve of the generator junction when an additional bias current is applied to the edge junction of the array. This effect was found to be due to the self-induced magnetic field produced by the edge current. This nonuniform field divides the array into domains each spanning several unit cells and each containing the same number of flux quanta. We report on experimental results obtained by low-temperature scanning electron microscopy (LTSEM) on the 1D array. The (1-3)- $\mu \mathrm{m}$ spatial resolution achieved by LTSEM enables us to image these domains in scanned measurements where the junctions in the array are heated sequentially. Computer simulations confirm the mechanism of the obtained images and the number of observed domains corresponds to the step position as predicted numerically.
\end{abstract}

\section{INTRODUCTION}

Rapid single-flux quantum (RSFQ) logic is one of the promising applications of superconducting electronic devices. ${ }^{1,2} \mathrm{~A}$ base element in the family of RSFQ logic circuits is the transmission line consisting of a parallel connection of resistively shunted Josephson junctions. A typical example with parallel dc current bias supply is shown in Fig. 1. The RSFQ transmission line may be employed to transfer single-flux quantum (SFQ) pulses between active elements, to amplify the magnetic field energy connected with the flux quantum, ${ }^{1}$ and to provide a time delay of fluxon propagation. $^{3-5}$

The one-dimensional (1D) array of Josephson junctions may also be used as a SFQ generator. When an additional bias current is applied to say the first junction ( $I_{1}$ in Fig. 1) it generates flux quanta, which can propagate down the transmission line. Since. $I_{1}$ is applied to the edge of the array we call it for short an edge current. As observed recently ${ }^{6}$ a particular series of steps shows up in the current-voltage (IV) curve of the "generator" junction. This effect was verified from computer simulations to be due to the self-induced magnetic field produced by the edge current. The underlying mechanism is that the nonuniform field divides the array into a number of domains each of which spans several unit cells and contains a given number of flux quanta. This is a dynamic state in a highly nonlinear system and the influence of differences in junction and array parameters on the domain stability is still unknown. The 1D array in many ways is analog to the long Josephson tunnel junction with regularly distributed inhomogeneities (in the limit where the distance between the inhomogeneities is larger than the Josephson penetration depth $\lambda_{J}$ ), and may be a model system for some of the flux flow junctions made with high- $T_{c}$ superconductors.

The main aim of this paper is to experimentally confirm and visualize the domains by using low-temperature scanning electron microscopy (LTSEM) and thus to determine which junctions are responsible for the different steps observed in the $I V$ curve.

\section{EXPERIMENTAL TECHNIQUE AND SAMPLE}

The LTSEM technique offers the possibility of a spatially resolved in situ investigation of superconducting tunnel junctions and integrated circuits. ${ }^{7-11}$ In that technique the top surface of the sample is scanned with an electron beam, while the bottom of the substrate carrying the junction is in intimate thermal contact with a bath of liquid helium. The main effect at the focus point $(x, y)$ of the electron beam is a local heating (0.1-10 $\mathrm{K}$ depending on the beam parameters) of the sample. The spatial resolution of about $1-3 \mu \mathrm{m}$ is determined by the thermal healing length of the sample con-

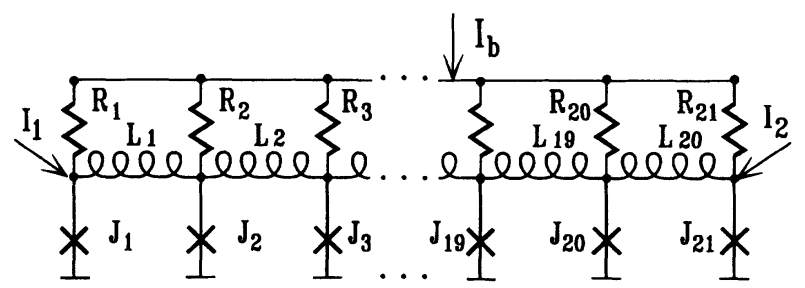

FIG. 1. Equivalent circuit of the 1D array of over-damped Josephson tunnel junctions $J_{1}-J_{21}$ (crosses). The relatively high identical resistances $R_{1}-R_{21}$ enforce a uniform distribution of the common bias current $I_{b}$. The edge currents $I_{1}$ and $I_{2}$ are fed directly to $J_{1}$ and $J_{21}$. The connecting inductances $L_{1}-L_{20}$ are equal. The array voltage $V$ is measured across one of the edge junctions. 


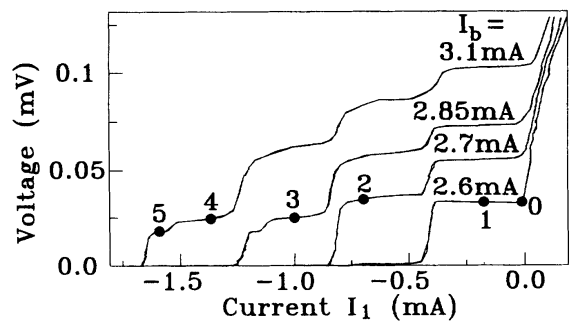

FIG. 2. Array voltage $V$ vs edge current $I_{1}$, measured for different values of $I_{b}$. The second edge current $I_{2}=0$. Marked points correspond to $I_{1}=-0.008 \mathrm{~mA}(0) ;-0.173 \mathrm{~mA}(1) ;-0.695 \mathrm{~mA}$

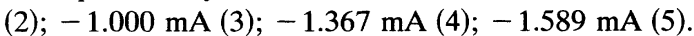

figuration. The beam power-typically $2.5 \mu \mathrm{W}$-can be adjusted over a wide range to ensure that the electron beam acts only as a passive probe on the sample. Scanning over the sample, the beam-induced signal, say the change in the voltage across the array, is simultaneously recorded with a digital imaging system.

Figure 1 shows the equivalent diagram of the circuit under investigation. The transmission line consists of a onedimensional array of twenty Josephson junctions $\left(J_{2}-J_{21}\right)$ connected in parallel and a generator junction $\left(J_{1}\right)$. The parameters of the circuit are the following: for junctions $J_{2}-J_{21}$ the critical current is $0.115 \mathrm{~mA}$, shunt resistance 1.2 $\Omega$, capacitance $0.5 \mathrm{pF}$, while for junction $J_{1}$ we have a critical current of $0.23 \mathrm{~mA}$, shunt resistance $0.6 \Omega$, and capacitance $1.0 \mathrm{pF}$. The higher critical current for junction $J_{1}$ is typical for RSFQ circuits. Each of the junctions are resistively shunted (externally) to provide a McCumber parameter, $\beta_{c} \approx 1$. The unit cell is a two-junction interferometer sharing its junctions with the neighboring cells. The inductances $L_{1}-L_{20}$ are $5 \mathrm{pH}$.

Standard trilayer $\mathrm{Nb}-\mathrm{Al}_{2} \mathrm{O}_{3}-\mathrm{Nb}$ technique was employed to fabricate the array. The junctions $J_{2}-J_{21}$ were circular with a diameter of $4 \mu \mathrm{m}$. The total length of the array was $1.5 \mathrm{~mm}$. Three bias currents, $I_{b}, I_{1}$ and $I_{2}$, were supplied to the array from high-resistance constant current sources: $I_{b}$ is a uniformly distributed current applied to the individual junctions through the resistances $R_{1}-R_{21}$. These resistances are ten times larger than the junction shunt resistances. The edge currents $I_{1}$ and $I_{2}$ are fed directly to junction $J_{1}$ and $J_{21}$, respectively. The array dc voltage $V$ and the electron beam-induced voltage signal $\Delta V(x, y)$ are measured across one of the edge junctions. $\Delta V(x, y)$ is detected by standard lock-in technique using the chopping frequency $(20 \mathrm{kHz})$ of the electron beam as reference. The output from the lock-in detector is fed to a digital imaging system. The underlying mechanism of $\Delta V(x, y)$, giving rise to the image of the above-mentioned domains, is a sequential heating of the individual Josephson junctions resulting in a reduction of their critical currents.

\section{EXPERIMENTAL RESULTS}

The experimental $V\left(I_{1}\right)$ curves obtained with $I_{2}=0$ for four different fixed values of $I_{b}$ are presented in Fig. 2. Qualitatively this result is similar to what we observed previously with a similar 21 -junction array. ${ }^{6}$ In the present experiment, however, the transmission line contains a generator

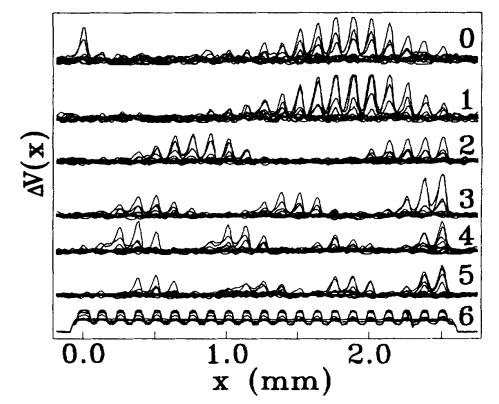

FIG. 3. LTSEM response $\Delta V(x)$ as a function of the beam position along the array. Images No. 0 -No. 5 are the signals proportional to the voltage increment of the steps at the marked points in Fig. 2. Each image consists of several line scans around the junction position (see text). Image No. 6 shows a usual SEM image of the sample. The images are vertically offset for clarity.

junction with two times higher critical current. From comparisons with the homogeneous array results we infer that the main effect of the generator junction is that it produces the small additional substeps barely visible on the $I V$ curves shown in Fig. 2. In both cases the bias current $I_{b}$ defines the velocity of fluxons moving through the array and thus defines the voltages of the (nearly) horizontal steps.

The main experimental results are shown in Fig. 3. Let us assume the array under investigation to be along the $x$ axis. In the experiment one usually has a slight misorientation between the scan line and the line connecting the centers of the junctions. This is the reason to present in Fig. 3 a series of line scans recorded with successive small offsets to new $y$ axis positions of the beam in order to completely cover all the junctions in the array. The images No. 0 through No. 5 in Fig. 3 correspond to the points with the same numbering in Fig. 2. The images in Fig. 3 are offset vertically for clarity. Image No. 6 in Fig. 3 is obtained from a usual scanning electron microscope (SEM) picture, and the maximum signal in these curves corresponds to the shunting resistances $R_{1}-R_{21}$; thus the positions of the midpoint of the shunting resistances coincide with the centers of the 21 junctions as they should.

In Fig. 3 one sees that the junction positions correspond to the maxima on images No. 0-No. 5 thus confirming that the signals really come from the functions. We also notice that the signals extend beyond the circumference of the junctions due to the heating of the area outside the junctions. The smooth envelope spanning several junction sites indicates that different junctions in the array play different roles in setting up the particular step voltages and that for different steps different groups of junctions are producing the LTSEM response. This is discussed in more detail below. It should be pointed out that the images were sensitive to parasitic magnetic flux trapped in the superconducting films. One procedure used to remove such trapped flux was to slowly scan the sample with high beam power.

\section{COMPUTER SIMULATIONS AND DISCUSSION}

In order to understand the nature of the recorded images a number of computer simulations were made. An important parameter for the calculations is the critical current reduction of a single junction produced by the electron beam. This 


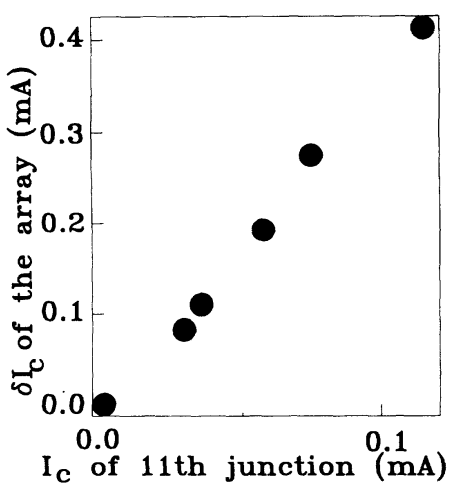

FIG. 4. Computer-simulated dependence of the reduction in the critical current of the array (critical value of $I_{b}$ ) as a function of the critical current of the 11th junction in the array.

parameter was determined experimentally by focusing the beam on the 11th junction and then measuring the reduction, $\delta I_{c}$, of the total critical current of the array. We found $\delta I_{c}=0.120 \mathrm{~mA}$, which is a little bit larger than the critical current $I_{c}=0.115 \mathrm{~mA}$ of a single junction. A numerical simulation, however, of $\delta I_{c}$ as a function of $I_{c}$ of the 11th junction gave the result shown in Fig. 4. Here we notice that $\delta I_{c}$ is about three times larger than the change in $I_{c}$ of a single junction. Based on this simulation result we can estimate the suppression of the critical current of a single junction to be $0.035 \mathrm{~mA}$, i.e., about $30 \%$. This effect shown in Fig. 4 is due to internal rearranging of currents and magnetic flux in the array, and may be related to the operation of the high- $T_{c}$ flux flow transistor. ${ }^{12}$ Alternatively it may be utilized as a kind of amplifier.

Figure 5 shows the simulated $I V$ curves obtained for the transmission line with parameters close to the experimental ones. As discussed below the only fitting parameter needed in order to reproduce most of the features of the experimental curves in Fig. 2 is a small additional edge current. We further simulated the change in the array voltage $\Delta V$ at the enumerated points in Fig. 5 as a function of the junction number for which the critical current was suppressed by $30 \%$. The results of these simulations are presented in Fig. 6. When these curves are compared with the experimental images in Fig. 3 there is a convincing overall resemblance.

Experimentally we found that the images were sensitive to the presence of trapped magnetic flux. This effect was also

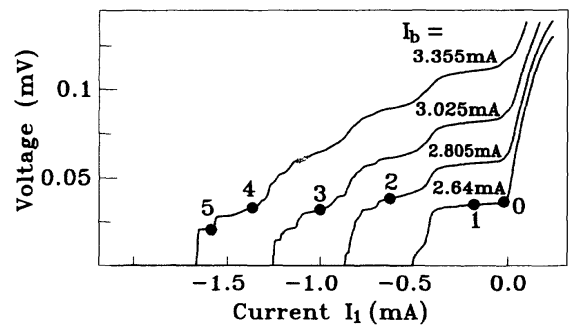

FIG. 5. Simulated array voltage $V$ vs edge current $I_{1}$ for different values of the uniform bias current $I_{b}$. The other edge current is set to $I_{2}=-0.0625 \mathrm{~mA}$ (see text). Marked points correspond to $I_{1}=-0.0125 \mathrm{~mA}(0) ;-0.175 \mathrm{~mA}(1) ;-0.625 \mathrm{~mA}(2) ;-1.000$ $\mathrm{mA}(3) ;-1.3625 \mathrm{~mA}(4) ;-1.5875 \mathrm{~mA}(5)$.

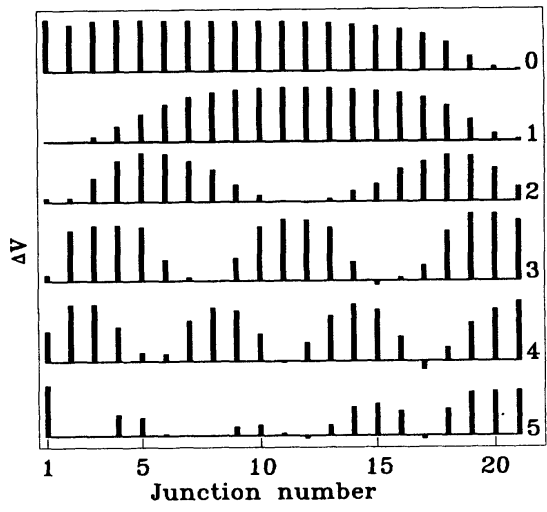

FIG. 6. Simulated LTSEM response $\Delta V$ (arbitrary units) as a function of the junction number. Images enumerated 0-5 show the voltage increment at the marked points in Fig. 5.

investigated numerically. In order to obtain the very best fit to Fig. 3 we had to introduce an additional edge current $I_{2}=-0.0625 \mathrm{~mA}$. Further simulation showed that this small current had no visible effects on the simulated $I V$ curve, and did not change the size of the domains with respect to the number of active junctions (see Fig. 6). Only minor details within the domains were modified.

With the good agreement between experiment and simulations we now look into the dynamics of the transmission line (see also Ref. 6). Figures 7(a)-7(f) show the instantaneous voltage across the individual junctions as a function of the junction number and time calculated with parameters corresponding to Fig. 6 . The voltage pulses appear whenever a fluxon is transferred from one cell to the next. As was described in detail in Ref. 6 the edge current causes a splitting of the array into domains each having predominantly the same number of fluxons per unit cell. It means that the magnetic field produced by the edge current does not penetrate into the array smoothly as in a long Josephson junction.

There are two kinds of domains in Figs. 7(a)-7(f): (i) The domains not reaching the edges are spatially symmetric; the junction in the middle of the domain (in the following called the leading junction) switches first, producing a fluxon and an antifluxon that in turn propagate in opposite directions. This means that the total fluxon number within a symmetrical domain is conserved at all times; (ii) The other kind of domains touch the edges; for those domains the position of the leading junction can be shifted from the center of the domain depending on the applied edge currents. Comparing the simulated images in Fig. 6 with the domain structures in Fig. 7 one may conclude that the experimental LTSEM images reproduce the position of the leading junctions. This is corroborated by the fact that the number of junctions within a given domain is approximately the same as seen in Fig. 7. Actually in the experiment the number of junctions in each domain may appear lower if the sensitivity of the LTSEM is reduced by, e.g., too low $e$-beam intensity.

The simulations depicted in Fig. 7 reveal one more feature. As was pointed out above, a generator junction with two times higher critical current creates substeps. Each step is associated with a definite number of domains and the substeps seem to originate in a redistribution of junctions among the domains [compare Figs. 7(e) and 7(f)]. The experiment 


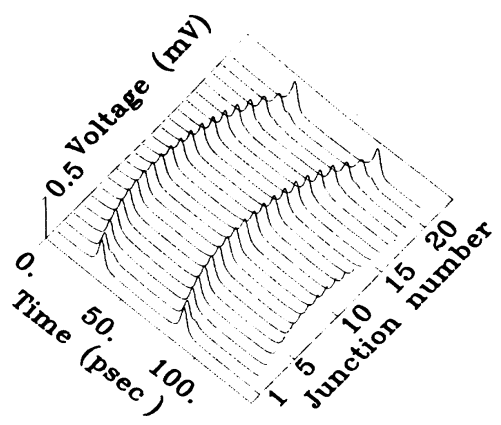

a)

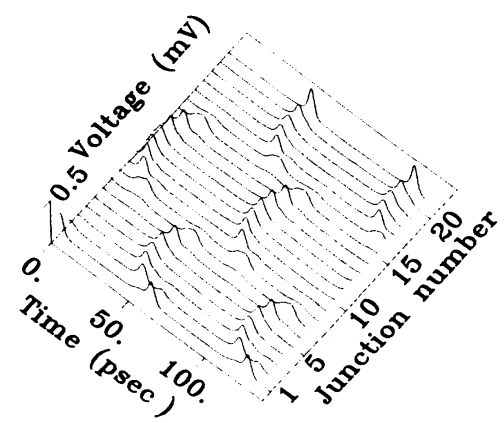

d)

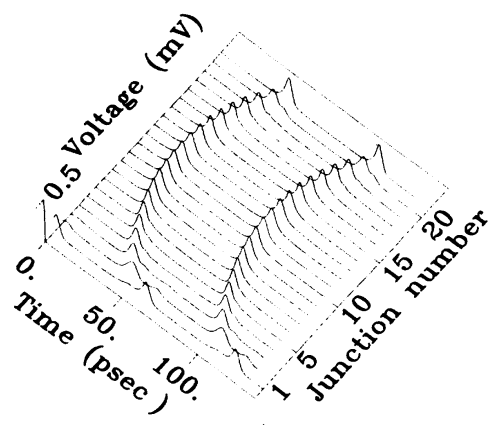

b)

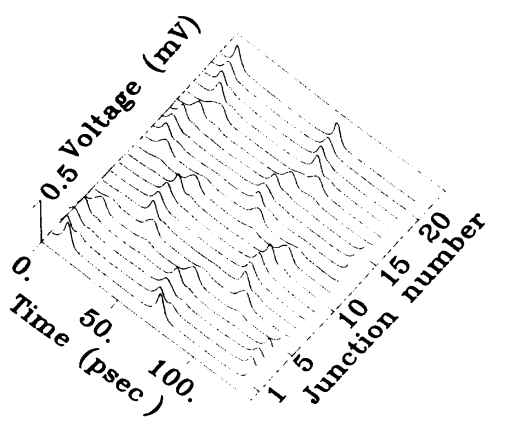

e)

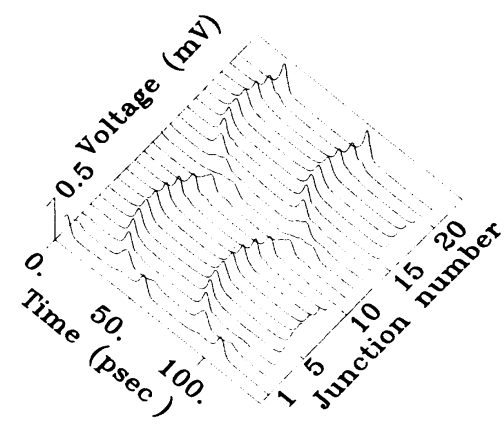

c)

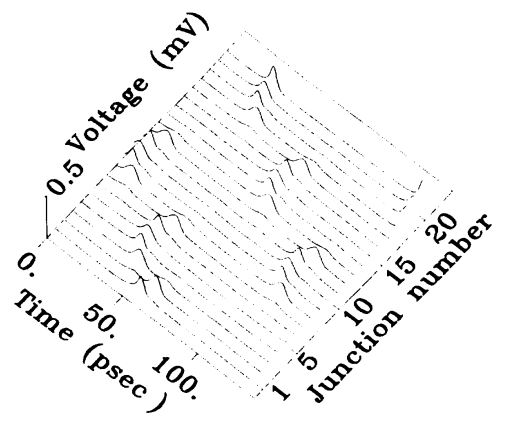

f)

FIG. 7. Simulated instantaneous voltage across the individual junctions as a function of the junction number and time. The edge current $I_{1}$ is injected to the first junction. Diagrams (a)-(f) correspond to the points marked $0-5$ in Fig. 5.

confirms this result when one compares the LTSEM image No. 4 with No. 5 in Fig. 3.

In summary, LTSEM is a powerful tool for a spatially resolved investigation of the dynamics of circuits based on shunted Josephson junctions. In this paper we reported on the possibility to recognize and visualize directly the dynamics of a one-dimensional parallel connected array of junctions. We envision many applications for this technique within areas such as RSFQ logic and high- $T_{c}$ based Josephson junction circuits.

\section{ACKNOWLEDGMENTS}

The authors thank Olga V. Kaplunenko, V. P. Koshelets, and S. Kovtonuk for help with the circuit fabrication, Susanne G. Lachenmann for assistance during the experiments, and G. M. Fischer, E. B. Goldobin, R. P. Huebener, M. I. Khabipov, Britt H. Larsen, and S. V. Shitov for fruitful discussions. The work was supported in parts by NORPAS, EU (CHRXCT 92-0068), NATO (L.G. 921040), and the Danish Research Academy.
${ }^{1}$ K. K. Likharev and V. K. Semenov, IEEE Supercond. 1, 13 (1991).

${ }^{2}$ V. K. Kaplunenko, M. I. Khabipov, V. P. Koshelets, K. K. Likharev, O. A. Mukhanov, I. L. Serpuchenko, and A. N. Vystavkin, IEEE Trans. Magn. MAG-25, 861 (1989).

${ }^{3}$ V. K. Kaplunenko, L. V. Filipenko, M. I. Khabipov, V. P. Koshelets, K. K. Likharev, S. V. Rylov, V. K. Semenov, and A. N. Vystavkin, IEEE Trans. Magn. MAG-27, 2464 (1991).

${ }^{4}$ V. K. Kaplunenko, M. I. Khabipov, and E. B. Goldobin, Supercond. Sci. Technol. 4, 674 (1991).

${ }^{5}$ E. B. Goldobin, V. K. Kaplunenko, M. I. Khabipov, and L. V. Filipenko, Cryogenics 32, 549 (1992).

${ }^{6}$ V. K. Kaplunenko, E. B. Goldobin, M. I. Khabipov, Britt H. Larsen, J. Mygind, and N. F. Pedersen, J. Appl. Phys. 74, 5854 (1993).

${ }^{7}$ R. P. Huebener, in Advances in Electronics and Electron Physics, edited by P. W. Hawkes (Academic, New York, 1988), Vol. 70, p. 1.

${ }^{8} \mathrm{~T}$. Doderer et al., in Nonlinear Superconductive Electronics and Josephson Devices, edited by G. Costabile, S. Pagano, N. F. Pedersen, and M. Russo (Plenum, New York, 1991), p. 353.

${ }^{9}$ T. Doderer, D. Hoffmann, R. P. Huebener, N. Kirchmann, C. A. Krülle, Susanne G. Lachenmann, D. Quenter, J. Schmidt, S. Stehle, J. Niemeyer, R. Pöpel, S. P. Benz, and P. A. A. Booi, IEEE Trans. Appl. Supercond. 3, 2724 (1993).

${ }^{10}$ D. Quenter, S. Stehle, T. Doderer, C. A. Krülle, R. P. Huebener, F. Müller, J. Niemeyer, R. Pöpel, T. Weimann, R. Ruby, and A. T. Barfknecht, Appl. Phys. Lett. 63, 2135 (1993).

${ }^{11}$ Susanne G. Lachenmann, T. Doderer, D. Hoffmann, R. P. Huebener, P. A. A. Booi, and S. P. Benz, Phys. Rev. B 50, 3158 (1994).

${ }^{12}$ J. S. Martens, V. M. Hietala, T. A. Plut, D. S. Ginley, G. A. Vawter, C. P. Tigges, M. P. Siegal, Julia M. Phillips, and S. Y. Hou, IEEE Trans. Appl. Supercond. 3, 2295 (1993). 